\title{
Pulmonary Mycobacterium kansasii infection: comparison of radiological appearances with pulmonary tuberculosis
}

\author{
A J Evans, A J Crisp, R B Hubbard, A Colville, S A Evans, I D A Johnston
}

\begin{abstract}
Background - A study was undertaken to determine if there are differences in the radiological appearances at presentation between pulmonary infections caused by Mycobacterium kansasii and Mycobacterium tuberculosis. Correct recognition of the organism has important implications with regard to initial therapy and contact tracing.
\end{abstract}

Methods - The initial chest radiographs of 28 patients with pulmonary $M$ kansasii infection were compared with those of 56 age, sex, and race matched patients with $M$ tuberculosis infection. All patients in both groups were culture positive and none was known to be HIV positive. The radiographs were analysed independently by two radiologists who were unaware of the causative organism.

Results - Radiographic abnormalities in patients with $M$ kansasii infection were more frequently unilateral and right side predominant, while those with tuberculosis more frequently involved a lower lobe. Air space shadowing involving more than one bronchopulmonary segment and pleural effusions were seen less frequently in $M$ kansasii infection (four of $28(14 \%)$ versus 30 of $56(54 \%)$ and none of 28 versus 15 of $56(27 \%)$ ). Cavitation (21 of $28(75 \%)$ versus 34 of $56(61 \%))$ was seen to a similar extent in patients with $M$ kansasii infection and in those with tuberculosis. Cavities tended to be smaller in patients with $M$ kansasii infection ( $p<0.01$ ).

Conclusions - Differences are seen in the radiographic appearances of pulmonary infection caused by $M$ kansasii and $M$ tuberculosis. These differences are not sufficient to allow a positive diagnosis on the basis of radiographic findings alone, but the presence of a pleural effusion or lower lobe involvement makes $M$ kansasii infection very unlikely.

(Thorax 1996;51:1243-1247)

Queen's Medical

Centre,

Nottingham NG7 2UH,

UK

Correspondence to:

Dr A J Evans,

Radiology Department,

City Hospital,

Nottingham NG5 IPB, UK

Received 2 February 1996

Returned to authors

9 April 1996

Revised version received

3 June 1996

Accepted for publication

18 June 1996
There is a wide geographical variation in the prevalence of the many species of non-tuberculous mycobacteria. ${ }^{1}$ Mycobacterium kansasi is the commonest species causing non-tuberculous mycobacterial pulmonary infection in the United Kingdom, ${ }^{2}$ Western Europe, and parts of the United States. ${ }^{3}$ In 1991 M kansasii accounted for $21 \%$ of all mycobacterial and $64 \%$ of non-tuberculous mycobacterial pulmonary infections in those of white race seen at our institution. ${ }^{4}$

Early clinical identification of $M$ kansasii infection would be helpful as it requires a different therapeutic regimen from $\mathrm{Myco}$ bacterium tuberculosis and contact tracing may be unnecessary. ${ }^{356}$ There have been several previous descriptions of the radiological appearances of $M$ kansasii infection but no attempt has been made to assess whether these differ from $M$ tuberculosis in comparable patients. We have therefore performed a blinded comparison of the radiological appearances of infections due to $M$ kansasii and $M$ tuberculosis in patients matched for age, sex, and race to determine if it is possible to make a specific diagnosis at presentation.

\section{Methods}

A review of the microbiological records held at University Hospital between 1978 and 1989 revealed that $M$ kansasii was isolated from the sputum of 50 patients. The chest radiographs of 34 of these patients were available for review, those of the other 16 patients having been destroyed. Each patient was matched (as closely as possible) for age, sex, and race with two control subjects (to enhance the power of the study) who had sputum cultures positive for $M$ tuberculosis within two years of the time $M$ kansasii was isolated. During this same period 690 patients had sputum cultures positive for $M$ tuberculosis.

Two radiologists, unaware of the infecting organism, independently evaluated the presenting chest radiographs in both groups. In three cases a discrepancy occurred which could not be resolved by consensus. In these cases a third independent opinion was obtained. The radiographs were assessed for the following features: previous or coexistent lung disease, site of abnormality, loss of lung volume, air space shadowing, circumscribed opacities and cavitation, bronchopulmonary spread, drainage area disease, local pleural disease, pleural effusions, lymphadenopathy, and evidence of a primary focus. Air space shadowing was classified as small if only one bronchopulmonary segment was involved and large if more than one segment was involved. Cavities were classified according to number, size, and the presence of air fluid levels. Circumscribed opacities were measured and numbered. 
Table 1 Radiological appearances of Mycobacterium kansasii and Mycobacterium tuberculosis pulmonary infections

\begin{tabular}{|c|c|c|c|c|c|}
\hline & $\begin{array}{l}M \text { kansasii } \\
(n=28)\end{array}$ & $\begin{array}{l}M \text { tuberculosis } \\
(n=56)\end{array}$ & OR & $95 \% C I$ & $p$ value \\
\hline $\begin{array}{l}\text { Unilateral } \\
\text { Predominant side }\end{array}$ & $20(71 \%)$ & $26(46 \%)$ & 2.60 & 1.02 to 6.58 & $0.045^{*}$ \\
\hline Left & $4(14 \%)$ & $14(25 \%)$ & 0.48 & 0.14 to 1.66 & 0.247 \\
\hline Right & $19(68 \%)$ & $24(43 \%)$ & 3.26 & 1.33 to 9.44 & $0.029^{*}$ \\
\hline Neither & $5(18 \%)$ & $18(32 \%)$ & 0.47 & 0.16 to 1.41 & 0.117 \\
\hline Lower lobe & $3(11 \%)$ & $24(43 \%)$ & 0.19 & 0.05 to 0.66 & $0.009^{*}$ \\
\hline Subapical & $24(86 \%)$ & $50(89 \%)$ & 0.69 & 0.16 to 2.94 & 0.619 \\
\hline Apical & $22(79 \%)$ & $40(71 \%)$ & 1.48 & 0.49 to 4.45 & 0.482 \\
\hline Circumscribed opacity & $4(14 \%)$ & $7(12.5 \%)$ & 1.14 & 0.33 to 3.90 & 0.831 \\
\hline Air space shadowing small & $13(46 \%)$ & $15(27 \%)$ & 2.08 & 0.86 to 5.03 & 0.103 \\
\hline Air space shadowing large & $4(14 \%)$ & $30(54 \%)$ & 0.20 & 0.07 to 0.6 & $0.004^{*}$ \\
\hline Volume loss & $16(57 \%)$ & $30(54 \%)$ & 1.154 & 0.47 to 2.86 & 0.758 \\
\hline Effusion & 0 & $15(27 \%)$ & & & $0.007^{* *}$ \\
\hline Drainage area disease & $11(39 \%)$ & $31(55 \%)$ & 0.55 & 0.22 to 1.35 & 0.189 \\
\hline Bronchopulmonary spread & $2(7 \%)$ & $8(14 \%)$ & 0.47 & 0.09 to 2.35 & 0.356 \\
\hline Local pleural disease & $16(57 \%)$ & $29(52 \%)$ & 1.24 & 0.50 to 3.10 & 0.644 \\
\hline Primary focus & $7(25 \%)$ & $13(23 \%)$ & 1.11 & 0.37 to 3.35 & 0.850 \\
\hline
\end{tabular}

*Statistically significant; **two tailed Fisher's exact test.

After reviewing the chest radiographs the medical records of the patients with sputum cultures positive for $M$ kansasii were obtained to determine if true pulmonary infection had occurred. Five patients did not satisfy the criteria of the American Thoracic Society for diagnosis of disease caused by non-tuberculous mycobacteria ${ }^{5}$ (multiple isolates, radiographic changes compatible with mycobacterial disease) and were excluded from the study. One patient who was HIV positive with AIDS was also excluded. The HIV status of the remaining patients was not known but no patients were members of a high risk group. The likelihood that any of these patients was HIV positive is extremely low as the prevalence of HIV infection in England and Wales outside London is currently about $0.01 \% .^{7}$

We therefore studied 28 patients with $M$ kansasii infection. All were white, $75 \%$ were men, their mean age was 60 years (range 3683 ), and the mean age at presentation was 57 years (range 30-83). Five patients had a history of tuberculosis, two had a history of Hodgkin's disease, two had rheumatoid arthritis (one of whom was taking corticosteroids), one had a history of alcohol abuse, and one had diabetes. The radiographic findings in these 28 patients were compared with the findings in 56 patients of mean age 57 years (range 26-87) who had $M$ tuberculosis infection.

Initial descriptive analysis was performed using the Statistical Package for Social Sciences (SPSS PC version 4.0). Matched case-control analysis for binary variables was carried out by conditional logistic regression using the EGRET statistical package. Where numbers were too small for conditional logistic regression Fisher's exact (two tailed) test was used. For number of cavities and maximal cavity size data were analysed using the $\chi^{2}$ test for linear trend (EPI-INFO version 6.0).

\section{Results}

The anatomical distribution of the radiological abnormalities differed between the two groups (table 1). Disease due to $M$ kansasii was confined to the upper lobes (apex/subapical) in 25 of the 28 patients $(89 \%)$ compared with 32 of the 56 patients $(57 \%)$ with $M$ tuberculosis
( $\mathrm{p}<0.01)$. Given the relative incidences of mycobacterial infections, the positive predictive value for $M$ tuberculosis infection rather than $M$ kansasii of lower lobe involvement was $93 \%$ (fig 1).

Of the three patients (11\%) with disease outside the upper lobes in the $M$ kansasii group, two were due to bronchopulmonary spread and in one with apical disease there was an accompanying bronchiectasis of the left lower lobe although infection of this lobe was not proven. Although upper lobe involvement was present in 50 of the 56 patients $(89 \%)$ with $M$ tuberculosis, in 19 of these disease was also present outside the upper lobes and in a further four patients disease was confined to the middle and/or lower lobes. $M$ kansasii was more commonly unilateral than $M$ tuberculosis (20 of 28 patients ( $71 \%$ ) compared with 26 of $56(46 \%)$; $\mathrm{p}=0.045)$. Disease due to $M$ kansasii was more commonly right side predominant than $M$ tuberculosis (19 of $28(68 \%$ ) compared with 24 of $56(43 \%) ; \mathrm{p}=0.029)$.

Further radiological findings are presented in table 1. Pleural effusions were not seen in the $M$ kansasii group but were present in 15 of

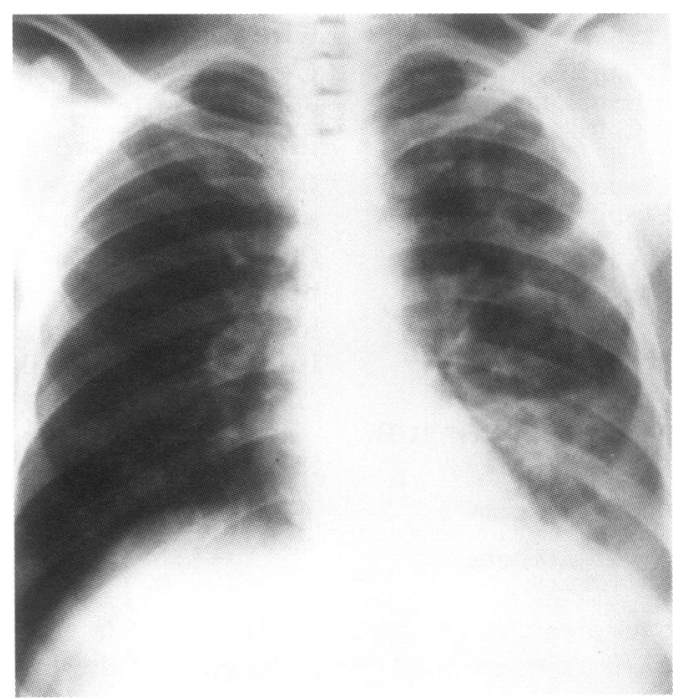

Figure 1 Chest radiograph of a patient with tuberculosis showing a left pleural effusion and left lower lobe involvement, features found less commonly in $M$ kansasii infection than in tuberculosis. 
Table 2 Cavities and evidence of previous or coexistent lung disease in Mycobacterium kansasii and Mycobacterium tuberculosis pulmonary infections

\begin{tabular}{lccc}
\hline & $\begin{array}{c}\text { M kansasii } \\
(n=28)\end{array}$ & $\begin{array}{c}\text { M tuberculosis } \\
(n=56)\end{array}$ & p value \\
\hline Cavities & $21(75 \%)$ & $34(61 \%)$ & $0.177^{*}$ \\
No. of cavities: & $7(33 \%)$ & $6(18 \%)$ & \\
1 & $4(19 \%)$ & $13(38 \%)$ & \\
$2-3$ & $2(10 \%)$ & $6(18 \%)$ & $0.986^{* *}$ \\
$4-5$ & $8(38 \%)$ & $9(26 \%)$ & \\
$>5$ & & & \\
Size of cavities: & $14(66 \%)$ & $10(29 \%)$ & \\
$<2 \mathrm{~cm}$ & $6(29 \%)$ & $16(47 \%)$ & \\
$2-3 \mathrm{~cm}$ & $1(5 \%)$ & $4(12 \%)$ & $0.006^{* *}$ \\
$3.1-6 \mathrm{~cm}$ & 0 & $4(12 \%)$ & $0.144^{* * *}$ \\
$>6 \mathrm{~cm}$ & 0 & $5(15 \%)$ & \\
Air fluid level & $6(21 \%)$ & $9(16 \%)$ & \\
Emphysema & $3(11 \%)$ & $2(4 \%)$ & \\
Bronchiectasis & & $3(5 \%)$ & \\
Coal worker's & & \\
$\quad$ pneumoconiosis & $1(4 \%)$ & $3(2)$ & \\
Asbestos exposure & $0(4 \%)$ & 0 & \\
Lymphoma & $1(4 \%)$ & & \\
Previous & & & \\
$\quad$ tuberculosis & $3(11 \%)$ & $3(5 \%)$ & \\
\hline
\end{tabular}

${ }^{*} \mathrm{OR}=2.13,95 \% \mathrm{CI} 0.71$ to $6.37 .{ }^{* *} \chi^{2}$ test for linear trend ***Fisher's exact test.

56 patients (27\%) with $M$ tuberculosis ( $\mathrm{p}<0.01$; fig 1). The positive predictive value of a pleural effusion for $M$ tuberculosis rather than $M$ kansasii in this series was therefore $100 \%$. In five (9\%) the effusion was the only abnormality present. Air space shadowing involving more than one bronchopulmonary segment was less common in the $M$ kansasii group (four of 28) than in the $M$ tuberculosis group (30 of 56, p <0.005), reflecting the more extensive involvement in the $M$ tuberculosis group. Miliary disease was seen in one patient with tuberculosis but was not seen in the $M$ kansasii group. Lymphadenopathy was not seen in either group.

Cavitation was present in similar proportions in both groups (table 2). There was a marked trend towards smaller cavities in the $M$ kansasii group (fig $2 ; \mathrm{p}<0.01$ ). Single thin walled cavities were present equally in both groups (fig 3). Air fluid levels within cavities were not seen in the $M$ kansasii group but were seen in five of 56 (9\%) of the $M$ tuberculosis group (fig 4).

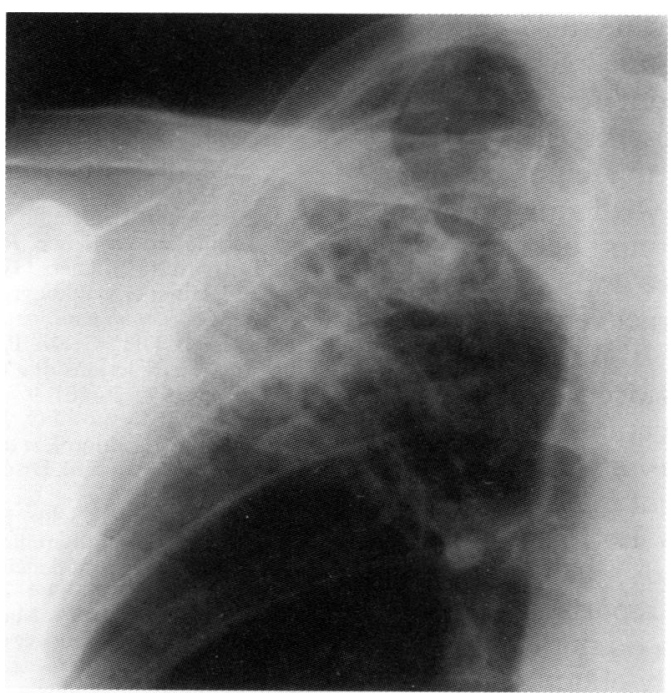

Figure 2 Chest radiograph of a patient with $M$ kansasii infection showing multiple small cavities in the right upper lobe.

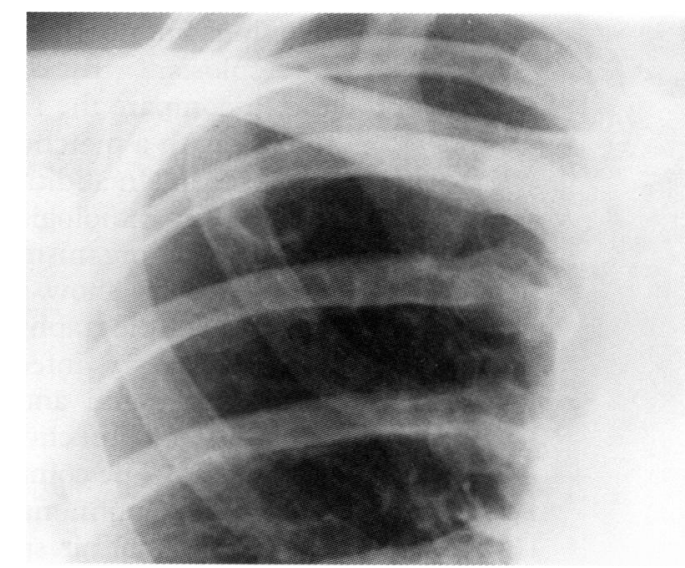

Figure 3 Chest radiograph of a patient with $M$ kansasii infection showing a solitary thin walled cavity with no surrounding consolidation in the right upper lobe.

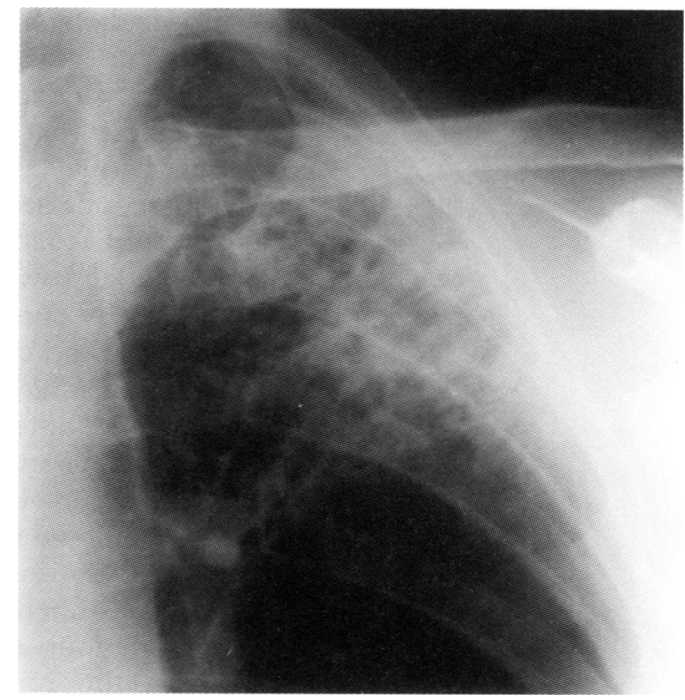

Figure 4 Chest radiograph of a patient with tuberculosis showing a large cavity in the left upper lobe containing an air fluid level, a feature not seen in $M$ kansasii infections.

\section{Discussion}

$M$ kansasii is the commonest non-tuberculous mycobacterium causing pulmonary infection in the $\mathrm{UK} .^{2}$ It requires a different therapeutic regimen from $M$ tuberculosis infection ${ }^{6}$ and, as human transmission rarely if ever occurs, ${ }^{8}$ contact tracing is unnecessary. ${ }^{3}$ The importance of $M$ kansasii infection and these differences from $M$ tuberculosis infection suggest that early clinical identification of $M$ kansasii would be helpful given the delay before bacteriological confirmation is available.

The radiographic features of pulmonary infections caused by $M$ kansasii have been reviewed by several authors. They have been variably reported as being indistinguishable from tuberculosis ${ }^{210}$ to having an appearance which is highly suggestive of $M$ kansasii infection $^{10-12}$ or one which is quite different from $M$ tuberculosis infection. ${ }^{1314}$ These variations in findings may, however, relate at least in part to differences in methods between these studies. Several of the studies ${ }^{101314}$ reported the radiographic features of non-tuberculous bacterial species as a single group, whereas others have described the features of $M$ kansasii 
infections but not directly compared them with tuberculosis. ${ }^{289} \mathrm{By}$ contrast, our study is the first to compare the radiological features of $M$ kansasii with a matched group of patients with tuberculosis. In addition, the radiographs were reviewed by radiologists who were unaware of the infecting organism.

Our results show that there is no pathognomonic radiographic appearance in $M$ kansasii pulmonary infection. The disease was usually unilateral and right sided. A similar distribution of infection was reported by Christensen et al. ${ }^{9}$ The commonest radiographic presentation of pulmonary $M$ kansasii infection was of minimal air space shadowing with associated cavitation confined to one or both upper lobes. In almost $90 \%$ of cases disease was confined to the upper lobes, as has generally been reported previously. ${ }^{8-10}$ In one study $50 \%$ of patients with non-tuberculous mycobacterial infection had disease outside the upper lobes, but the study included four nontuberculous mycobacterial species. ${ }^{13}$ Recent evidence suggests that the radiographic features of the individual non-tuberculous mycobacterial infections are diverse. ${ }^{15}$

Previous studies have reported cavitation in $84-96 \%$ of patients with $M$ kansasii pulmonary infections. ${ }^{2916}$ In our study $75 \%$ of patients with $M$ kansasii had cavitation and neither we nor Christensen et $a l^{16}$ found the frequency of cavitation to be different from tuberculosis. Two other previous studies ${ }^{1314}$ reported a much lower incidence (38-43\%) of cavitating disease in non-tuberculous mycobacterial infection. These studies, however, included $M$ kansasii with other non-tuberculous species and their patient populations are therefore not comparable with those in the studies reviewed above. Zvetina et $a l^{11}$ reported that a single thin walled cavity with little surrounding parenchymal disease was highly likely to be due to $M$ kansasii infection. We found solitary cavities more frequently, though not significantly so, in patients with $M$ kansasii infection, as did Christensen et al. ${ }^{16}$ In clinical practice, however, because tuberculosis is five times more common than $M$ kansasii infection, a solitary cavity is much more likely to be due to $M$ tuberculosis infection than to $M$ kansasii infection. There are, however, significant differences in the appearances of cavities in patients with $M$ kansasii infection and those with tuberculosis. Patients with $M$ kansasii generally had smaller cavities and air fluid levels did not occur. Overall, however, the number, size and appearance of cavities was not characteristic enough to make a positive diagnosis of $M$ kansasii infection.

Pleural effusions rarely occur in non-tuberculous mycobacterial infections. ${ }^{516}$ Indeed, in our study no pleural effusions were seen with $M$ kansasii but were present in nearly a third of patients with tuberculosis. Local or contiguous pleural disease was found equally in the two infections. Chapman ${ }^{17}$ reported that nontuberculous mycobacteria do not form a primary complex. In our study about a quarter of patients with either infection were found to have a primary focus but, for those with $M$ kansasii infection, this may be due to previous
$M$ tuberculosis infection. Certainly, three of the seven patients had previously been diagnosed and treated for pulmonary tuberculosis. The association of non-tuberculous mycobacterial infections with previous mycobacterial disease has been reported. ${ }^{5}$ Two previous studies ${ }^{1415}$ have reported a more disseminated infection with little associated cavitation in a group of patients with non-tuberculous mycobacterial infection. Both studies included several species of non-tuberculous mycobacteria in addition to $M$ kansasii and, as the radiographic appearances in these individual infections may be diverse,$^{15}$ these patients are not comparable with those in this or other previous studies. ${ }^{28-1016}$

Disseminated disease due to $M$ kansasii infection is rarely seen but has been reported in immunosuppressed patients, ${ }^{18}$ including those with AIDS, 19 though more than $95 \%$ of disseminated non-tuberculous mycobacterial disease in AIDS is due to the Mycobacterium avium intracellulare complex. ${ }^{1220}$

This study shows that there are some significant differences in the radiological appearances in patients with $M$ kansasii pulmonary infections when compared with matched controls with pulmonary tuberculosis. Lower lobe involvement or a pleural effusion make $M$ kansasii infection very unlikely. However, those features that are more common in $M$ kansasii infection do not display radiological differences that are significantly great to allow pulmonary $M$ kansasii infection to be positively differentiated from tuberculosis.

1 Buckner CB, Leithiser RE, Walker CW, Allison JW. The changing epidemiology of tuberculosis and other mycochanging epidemiology of tuberculosis and other mycothe radiologist. $A \mathcal{F}$ 1991;156:255-64.

2 Banks J, Hunter AM, Campbell IA, Jenkins PA, Smith AP. Pulmonary infection with Mycobacterium kansasii in Wales, 1970-79: review of treatment and response. Thorax 1983; 38:271-4.

3 Davies PDO. Infection with Mycobacterium kansasii. Thorax 1994;49:435-6.

4 Colville A, Baker M. Culture positive pulmonary mycobacterial disease in Nottingham 1979-1991: an increasing proportion of non-tuberculous infection. Thorax 1992;47: proport 875 .

5 American Thoracic Society. Diagnosis and treatment of disease caused by non-tuberculous mycobacteria. $\mathrm{Am} \mathrm{Rev}$ Respir Dis 1990;142:940-53.

6 Research Committee, BTS. Mycobacterium kansasii pulmonary infection: a prospective study of the results of nine months of treatment with rifampicin and ethambutol. Thorax 1994;49:442-5.

7 Communicable Disease Report. Unlinked anonymous monitoring of HIV prevalence in England and Wales: monitoring of HIV prevalence in

8 Lillo M, Orengo S, Cernock P, Harris RL. Pulmonary and disseminated infection due to Mycobacterium kansasii: a decade of experience. Rev Infect Dis 1990;12:760-7.

9 Christensen EE, Dietz GW, Ahn CH, Chapman JS, Murry RC, Hurst GA. Radiographic manifestations of pulmonary Mycobacterium kansasii infections. AfR 1978;131:985-93.

10 Anderson DH, Grech P, Townshend RH, Jepchott AE Pulmonary lesions due to opportunist mycobacteria. Clin Radiol 1975;26:461-9.

11 Zvetina JR, Demos TC, Maliwan N, van Drunen M, Frederick W, Lentino J, et al. Pulmonary cavitations in Mycoderick W, Lentino , et al. Pulmonary cavitations in Myco-
bacterium kansasii. Distinction from $M$ tuberculosis. AfR bacterium kansasii.

12 Horsburgh CR, Selik RM. The epidemiology of disseminated nontuberculous mycobacterial infection in the acquired immunodeficiency syndrome (AIDS). Am Rev Respir Dis 1989;139:4-7.

13 Albelda SM, Kern JA, Marinelli DL, Miller WT. Expanding spectrum of pulmonary disease caused by non-tuberculous mycobacteria. Radiology 1985;157:289-96.

14 Woodring JH, Vandiviere HM. Pulmonary disease caused by non-tuberculous mycobacteria. $\mathcal{F}$ Thorac Imaging 1990 ; 5:64-76.

15 Evans AJ, Crisp AJ, Colville A, Evans SA, Johnston IDA Pulmonary infections caused by Mycobacterium malmoense 
and Mycobacterium tuberculosis: comparison of radiographic

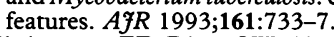

16 Christensen EE, Dietz GW, Ahn $\mathrm{CH}$, Chapman IS, Murry $\mathrm{RC}$, Anderson J, et al. Initial roentgenographic manifestations of pulmonary Mycobacterium tuberculosis, $M$ kan sasii and $M$ intracellularis. Chest 1981;80:132-6.

17 Chapman JS. The atypical mycobacteria and human mycobacterioses. New York: Plenum Press, 1977: 60-2.

18 Lichtenstein IH, MacGregor RR. Mycobacterial infections in renal transplant recipients: report of five cases and review of the literature. Rev Infect Dis 1983;5:216-26.

19 Sherer R, Sable R, Sonnenberg M. Disseminated infection with Mycobacterium kansasii in the acquired immunodeficiency syndrome. Ann Intern Med 1986;105:710-2.

20 Marinelli DL, Albelda SM, Williams TM, Kern JA, Iozzo RV, Miller WT. Non-tuberculous mycobacterial infection in AIDS: clinical, pathologic, and radiographic features. Radiology 1986;160:77-82. 